\title{
NUMERICAL SOLUTION OF EUROPEAN AND AMERICAN OPTION WITH DIVIDENDS USING FINITE DIFFERENCE METHODS
}

\author{
Kedar Nath Uprety and Ganesh Prasad Panday \\ Central Department of Mathematics, Tribhuvan University, Kirtipur, Kathmandu, Nepal
}

\begin{abstract}
Numerical methods form an important part of the pricing of financial derivatives where there is no closed form analytical formula. Black-Scholes equation is a well known partial differential equation in financial mathematics. In this paper, we have studied the numerical solutions of the Black-Scholes equation for European options (Call and Put) as well as American options with dividends. We have used different approximate to discretize the partial differential equation in space and explicit (Forward Euler's), fully implicit with projected Successive Over-Relaxation (SOR) algorithm and Crank-Nicolson scheme for time stepping. We have implemented and tested the methods in MATLAB. Finally, some numerical results have been presented and the effects of dividend payments on option pricing have also been considered.
\end{abstract}

Keywords: Black-Scholes model with dividends; Call and put options; Finite difference schemes.

\section{INTRODUCTION}

The famous Black-Scholes equation is an effective model for option pricing. It was named after the pioneers Black, Scholes and Merton who suggested it $1973^{1,3}$ and received in 1997 the Nobel Prize in Economics for their discovery $^{2}$. Mathematically, it is a final value problem for a second order parabolic equation. A concise derivation of the Black-Scholes equation can be found in ${ }^{5}$.

Black and Scholes (1973) proposed a valuation model for an European option, a contract that allows the holder of the option to exercise the right to buy or sell stocks at the expiration date. Unlike European options, where the payoff is determined by the price of the underlying asset at the exercise date, another primary type of options called American options give the holder the right to early exercise the options at any time before the expiration date. The American option valuation problem can be viewed as a free-boundary problem, that is, there exists an unknown boundary dependent on time, that sets the line between early exercising and holding the option. Since an American option offers the holder greater rights than an European option, it usually has a larger value. The explicit formula for European options no longer works for American options. Our approach to obtain the result of the American option valuation problem is to rewrite the problem in a linear complementarity form. We then solve it using finite difference methods and the projected Successive Over-Relaxation (SOR) algorithm. The use of the linear complementarity form has a great advantage in that the free boundary points are implicitly included in a single constraint. Those points no longer need to be explicitly mentioned, which facilitates the computation of the option values.

\section{The Black-Scholes Equation}

We consider only American and European call option. Similar treatment can be done for the American and European put option. The value of a option is denoted by $V$ and depends on the current market price of the underlying asset, $S$, and the remaining time $t$ until the option expires: $V=V(S, t)$. The Black-Scholes equation is a backward-in-time parabolic equation and posed on a time dependent domain.

With dividend payments:

$$
\frac{\partial V}{\partial t}+\left(r-D_{0}\right) S \frac{\partial V}{\partial S}+\frac{1}{2} \sigma^{2} S^{2} \frac{\partial^{2} V}{\partial S^{2}}-r V=0
$$

for

$$
0<S<S_{f}(t), 0 \leq t<T,
$$

With no dividend payments:

for

$$
\frac{\partial V}{\partial t}+r S \frac{\partial V}{\partial S}+\frac{1}{2} \sigma^{2} S^{2} \frac{\partial^{2} V}{\partial S^{2}}-r V=0
$$

$$
0<S, 0 \leq t<T \text {, }
$$

where $\sigma$ denotes the annual volatility of the asset price, $r$ the risk-free interest rate and $T$ is the expiry date $(t=0$ means today). We assume that dividends are paid with a continuous yields of constant level $D_{0}>0$. Note that we have to include the payment of dividends. Otherwise, for $D_{0}=0$ early exercise does not make sense and the American call would be equivalent to the European one . $^{3}$.

\section{European Option}

A European call (put) option gives the holder the right but not the obligation to buy (sell) the underlying asset with an initial price $S$; at a given maturity date $T$ and for a fixed price $E$; called the strike price. Let the price of European call (put) option be denoted by $C(P)$ : These notations will be used throughout our work to denote the European call and put option. The payoff of a European call at maturity time $T$ is,

$$
C=\max \left(S_{T}-E, 0\right) .
$$

If $S_{T}<E$, the call will be worthless and the holder will not exercise the right. The payoff of a European put is, 


$$
P=\max \left(E-S_{T}, 0\right) .
$$

If $S_{T}>E$, the put will be worthless and the holder will not exercise the right. The call - put parity is the relationship between a European call and put, given by,

$$
C+E e^{-r t}=P+S .
$$

where $r$ denotes the risk free interest rate and $\mathrm{S}$ the initial stock price.

\section{American Options}

American call (put) option gives to its holder the right but not the obligation to buy (sell) the underlying asset at any time $t(0<t<T)$, up to maturity date $T$, for a strike price $E$. Let the price of the American call (put) option be denoted by $C(P)$. These notations will be used throughout our work to denote the American call and put option. The payoff of an American call at maturity time $T$ is,

$$
C=\max \left(S_{T}-E, 0\right)
$$

The payoff of an American put is,

$$
P=\max \left(E-S_{T}, 0\right)
$$

The price boundary and put-call parity for the American option is given by,

$$
S-E \leq C-P \leq S-E e^{-r t}
$$

\section{Free Boundary Problem}

At $S=0$ the option is worthless. Note that we need two conditions at the free boundary $S=S_{f}(t)$. One condition is necessary for the solution of (1) and other is needed for determining the position of the free boundary $S_{f}(t)$ itself. The condition in $V\left(S_{f}(t), t\right)=S_{f}(t)-E$ for $0 \leq t \leq$ $T$ and $V_{S}\left(S_{f}(t), t\right)=1$ for $0 \leq t \leq T$ ('value matching' condition) is the continuity of the mapping $S \rightarrow V(S, t)$ since $V(S, t)=(S-E)^{+}=S-E$, in the exercise region $S$ $\geq S_{f}(t)$. At $S=S_{f}(t)$ one requires additionally that $\mathrm{V}(\mathrm{S}, \mathrm{t})$ touches the payoff function tangentially ('high contact condition'), i.e. the function $S \rightarrow \partial V(S, t) / \partial S$ should be continuous at $S=S_{f}(t)$. The conditions $V\left(S_{f}(t), t\right)=$ $S_{f}(t)-E$ for $0 \leq t \leq T$ and $V_{S}\left(S_{f}(t), t\right)=1$ for $0 \leq t \leq T$ are jointly referred as the 'smooth-pasting conditions'. Note that the later condition can be derived from an arbitrage argument ${ }^{4,5}$.

Since American options can be exercised at any time, we have the a priori bound

$$
V(S, t) \geq(S-E)^{+}, \quad S \geq 0, \quad 0 \leq t \leq T .
$$

If $V(S, t)<(S-E)^{+}$for one value $S>E$ and $t \leq T$ then the purchase of a call for $\mathrm{V}$ and the immediate exercise of this option to buy the underlying asset for $\mathrm{E}$ (although its value is $\mathrm{S}$ ) would lead to an instantaneous risk-free profit of $S-V-E>0$, in violation to the noarbitrage principle. Of course, this reasoning ignores transaction costs. For American options, when $V>S-$ $E$, meaning it is optimal to hold the option, the BlackScholes equation holds. Otherwise, $V=S-E$; it is optimal to exercise the option. The two relationships can be combined into one inequality for the BlackScholes equation.

$$
\frac{\partial V}{\partial t}+\left(r-D_{0}\right) S \frac{\partial V}{\partial S}+\frac{1}{2} \sigma^{2} S^{2} \frac{\partial^{2} V}{\partial S^{2}}-r V \leq 0
$$

where $\mathrm{V}(\mathrm{S}, \mathrm{t})$ is the value of a call option, $t \in[0, T]$. Another two constraints coming from no arbitrage assumption are that the option value has to be continuous since holders can profit from exercising when the asset price reaches the value of the discontinuity and that the change of the option value should also be continuous.

Let $x= \pm 1$ be the end points of the string; $u(x)$ be the string displacement; $f(x)$ be the height of the obstacle. We do not have knowledge on the exact region of contact between the string and the obstacle. We only know the string must either be above or on the obstacle, and the string and the slope of the string have to be continuous. The free boundary is the set of points $P\left(x=x_{p}\right)$ and $Q\left(x=x_{Q}\right)$, the points that define the contact region. Since the contact region concaves down, $u=f$ and $u^{\prime \prime}<0$, while $u>f$ and $u^{\prime \prime}=0$ when the string is above the obstacle. We also assume that

$$
f( \pm 1)<0, f(x)>0 \text { for some }-1<x<1, f^{\prime \prime}<0
$$

to ensure there exists only one contact region.

The obstacle problem is then equivalent to finding $u(x)$ and the points $\mathrm{P}, \mathrm{Q}$ such that

$$
\begin{aligned}
& u(-1)=0, u(1)=0 \\
& u^{\prime \prime}=0,-1<x<x_{P}, x_{Q}<x<1 \\
& u\left(x_{P}\right)=f\left(x_{P}\right), u^{\prime}\left(x_{P}\right)=f^{\prime}\left(x_{P}\right) \\
& u\left(x_{Q}\right)=f\left(x_{Q}\right), u^{\prime}\left(x_{Q}\right)=f^{\prime}\left(x_{Q}\right) \\
& u(x)=f(x), x_{P}<x<x_{Q} .
\end{aligned}
$$

One approach to solve this problem is to first write it in the following linear complementarity form

$$
u^{\prime \prime} .(u-f)=0,-u^{\prime \prime} \geq 0,(u-f) \geq 0,
$$

where $u(-1)=u(1)=0$, and $\mathrm{u}, u^{\prime}$ are continuous. This transformation does not explicitly include the free boundary points. Instead, the free boundary problem is implicitly incorporated in the constraint $u \geq f$. We can then use numerical techniques such as iterative methods to solve (12).

\section{Transformed Linear Black-Scholes Equation}

Here we transform (1) problem from the original $(S, t)$ variables to $(x, \tau)$ into a pure diffusion equation, where $x$ and $\tau$ refer to the following transformation. Let

and

$$
x=\ln \left(\frac{S}{E}\right)
$$

$$
t=T-\left(\frac{\tau}{\left(\sigma^{2} / 2\right)}\right)
$$

and

$$
v(x, \tau)=\frac{1}{E} V(S, t)
$$

Now

$$
\begin{gathered}
\frac{\partial V}{\partial t}=\frac{\partial V}{\partial \tau} \frac{\partial \tau}{\partial t}=-\frac{\sigma^{2}}{2} E \frac{\partial v}{\partial \tau} \\
\frac{\partial V}{\partial S}=E \frac{\partial v}{\partial S}=\frac{E}{S} \frac{\partial v}{\partial x},
\end{gathered}
$$


and

$$
\frac{\partial^{2} V}{\partial S^{2}}=E \frac{\partial^{2} v}{\partial S^{2}}=\frac{E}{S^{2}}\left[\frac{\partial^{2} v}{\partial x^{2}}-\frac{\partial v}{\partial x}\right]
$$

Substituting these all derivatives in (1) we have $-\frac{\sigma^{2}}{2} E \frac{\partial v}{\partial \tau}+\left(r-D_{0}\right) E \frac{\partial v}{\partial x}+\frac{\sigma^{2}}{2} E\left[\frac{\partial^{2} v}{\partial x^{2}}-\frac{\partial v}{\partial x}\right]-r E v=0$ and a final multiplication by $-\frac{2}{\sigma^{2} E}$ then gives

$$
\frac{\partial v}{\partial \tau}=\frac{\partial^{2} v}{\partial x^{2}}+\left(\frac{2\left(r-D_{0}\right)}{\sigma^{2}}-1\right) \frac{\partial v}{\partial x}-\frac{2 r}{\sigma^{2}} v
$$

Let $\frac{2 r}{\sigma^{2}}=K_{\text {and }} \frac{2\left(r-D_{0}\right)}{\sigma^{2}}=K^{\prime}$ in (13) can be written as

$$
\frac{\partial v}{\partial \tau}=\frac{\partial^{2} v}{\partial x^{2}}+\left(K^{\prime}-1\right) \frac{\partial v}{\partial x}-K v
$$

Now consider $\alpha=\frac{-1}{2}\left(K^{\prime}-1\right), \beta=\frac{-1}{4}\left(K^{\prime}-1\right)^{2}-K$, to get $\beta=-\alpha^{2}-K$, and then

$$
\begin{aligned}
v(x, \tau) & =e^{\alpha x+\beta \tau} u(x, \tau) \\
\frac{\partial v}{\partial \tau} & =e^{\alpha x+\beta \tau} \beta u+\frac{\partial u}{\partial \tau} e^{\alpha x+\beta \tau} \\
& =e^{\alpha x+\beta \tau}\left[\beta u+\frac{\partial u}{\partial \tau}\right],
\end{aligned}
$$

and

$$
\begin{gathered}
\frac{\partial v}{\partial x}=e^{\alpha x+\beta \tau}\left[\alpha u+\frac{\partial u}{\partial x}\right], \\
\frac{\partial^{2} v}{\partial x^{2}}=e^{\alpha x+\beta \tau}\left[\alpha^{2} u+2 \alpha \frac{\partial u}{\partial x}+\frac{\partial^{2} u}{\partial x^{2}}\right],
\end{gathered}
$$

Substituting these into equation (14) and dividing by $e^{\alpha x+\beta \tau}$ we get

$$
\begin{aligned}
\beta u+\frac{\partial u}{\partial \tau}=\left[\alpha^{2} u+2 \alpha \frac{\partial u}{\partial x}+\frac{\partial^{2} u}{\partial x^{2}}\right]+ \\
\left(K^{\prime}-1\right)\left[\alpha u+\frac{\partial u}{\partial x}\right]-K u,
\end{aligned}
$$

implies

$$
\begin{aligned}
& \frac{\partial u}{\partial \tau}=\frac{\partial^{2} u}{\partial x^{2}}+\left(2 \alpha+K^{\prime}-1\right) \frac{\partial u}{\partial x}+ \\
&\left(\alpha^{2}-\beta^{2}+\alpha\left(K^{\prime}-1\right)-K\right) u
\end{aligned}
$$

and finally we get,

$$
u_{\tau}=u_{x x},-\infty<x<\infty, \tau>0,
$$

which is a diffusion equation with initial condition $u(x, 0)=v(x, 0) e^{-\alpha x}=\max \left(e^{\frac{1}{2}\left(K^{\prime}+1\right) x}-e^{\frac{1}{2}\left(K^{\prime}-1\right) x}, 0\right)$

Observe that with the transformation above, the dividend term does not appear explicitly in equation (15).

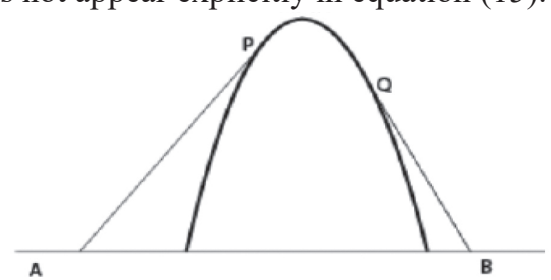

(a) The classical obstacle problem: the string is held fixed at A and B and must pass smoothly over the obstacle in between.(Wilmott,1995)

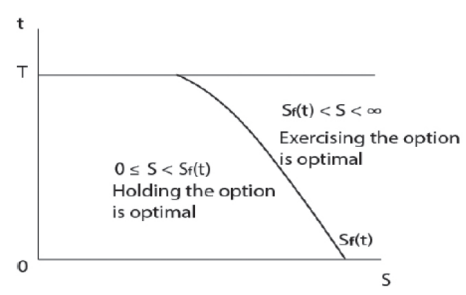

(b) Free boundary problem.(Wilmott,1995)

Figure. American Options
For European Call

The boundary condition for the European call option

$$
\begin{gathered}
u(x, 0)=g(x, 0), \\
\lim _{x \rightarrow-\infty} u(x, \tau)=0, \\
\lim _{x \rightarrow \infty} u(x, \tau)=g(x, \tau),
\end{gathered}
$$

where

$$
\begin{gathered}
g(x, \tau)=e^{\left(\frac{1}{4}\left(K^{\prime}-1\right)^{2}+K\right) \tau} \max \left(\left(e^{\frac{1}{2}\left(K^{\prime}+1\right) x}-e^{\frac{1}{2}\left(K^{\prime}-1\right) x}\right), 0\right) \\
g(x, \tau)=e^{-\beta \tau} \max \left(\left(e^{(-\alpha+1) x}-e^{-\alpha x}\right), 0\right) \\
g(x, \tau)=e^{-\beta \tau}\left(e^{(-\alpha+1) x}-e^{-\alpha x}\right)^{+} \\
g(x, \tau)=e^{-\alpha x-\beta \tau}\left(e^{x}-1\right)^{+}
\end{gathered}
$$

\section{Linear Complementarity Form for American Option}

The obstacle problem for American call options with dividends is equivalent to finding $u(x, \tau)$ and the unknown optimal exercise boundary $x_{f}(\tau)$ such that

$$
\begin{aligned}
& u_{\tau}=u_{x x}, \text { for } x \leq x_{f}(\tau) \\
& u(x, \tau)=g(x, \tau) \text { for } x>x_{f}(\tau),
\end{aligned}
$$

with boundary conditions

$$
\begin{gathered}
u(x, 0)=g(x, 0), \\
\lim _{x \rightarrow-\infty} u(x, \tau)=0, \\
\lim _{x \rightarrow \infty} u(x, \tau)=g(x, \tau),
\end{gathered}
$$

where

$$
\begin{gathered}
g(x, \tau)=e^{\left(\frac{1}{4}\left(K^{\prime}-1\right)^{2}+K\right) \tau} \max \left(\left(e^{\frac{1}{2}\left(K^{\prime}+1\right) x}-e^{\frac{1}{2}\left(K^{\prime}-1\right) x}\right), 0\right) \\
g(x, \tau)=e^{-\beta \tau} \max \left(\left(e^{(-\alpha+1) x}-e^{-\alpha x}\right), 0\right) \\
g(x, \tau)=e^{-\beta \tau}\left(e^{(-\alpha+1) x}-e^{-\alpha x}\right)^{+} \\
g(x, \tau)=e^{-\alpha x-\beta \tau}\left(e^{x}-1\right)^{+}
\end{gathered}
$$

We also have the constraint

$$
u=(x, \tau) \geq g(x, \tau)
$$

Since we will be focusing on numerical solutions using finite difference methods, we will restrict the problem to a finite interval. Therefore, we consider the problem only for $\mathrm{x}$ in the interval $\left[\mathrm{x}^{-}, \mathrm{x}^{+}\right]$, where $\mathrm{x}^{-}$is a large negative number and $\mathrm{x}^{+}$is a large positive number. Hence, the boundary conditions are

$$
u\left(x^{-}, \tau\right)=0, \quad u\left(x^{+}, \tau\right)=g\left(x^{+}, \tau\right) .
$$

Now, rewriting in a linear complementarity form, we obtain

$$
\left(\frac{\partial u}{\partial \tau}-\frac{\partial^{2} u}{\partial x^{2}}\right)(u(x, \tau)-g(x, \tau))=0
$$

with two constraints

$$
\frac{\partial u}{\partial \tau}-\frac{\partial^{2} u}{\partial x^{2}} \geq 0,(u(x, \tau)-g(x, \tau)) \geq 0,
$$

with the initial condition $v(x, 0)=g(x, 0)$ and the boundary conditions 


$$
\begin{array}{r}
u\left(x^{-}, \tau\right)=g\left(x^{-}, \tau\right)=0, \\
u\left(x^{+}, \tau\right)=g\left(x^{+}, \tau\right) .
\end{array}
$$

The above transformation is helpful because the diffusion equation is more straightforward and less cluttered than the Black-Scholes equation. It is much easier to find numerical solutions of the diffusion equation and then to convert these into numerical solutions of the BlackScholes equation through a change of variables than to numerically solve the Black-Scholes equation directly. Thus to obtain the numerical result of American option value, our approach is to solve

$$
\frac{\partial u}{\partial \tau}-\frac{\partial^{2} u}{\partial x^{2}}=0
$$

and make sure

$$
u(x, \tau)-g(x, \tau) \geq 0
$$

\section{Finite Difference Methods}

The finite difference methods attempt to solve Black Scholes Partial differential equation by approximating the differential equation over the area of integration by a system of algebraic equations. They are a means of obtaining numerical solutions to Partial differential equations.They also constitute a very powerful and therefore flexible technique that is capable of generating accurate numerical solutions to PDEs arising in financial and other physical sciences. The most common finite difference methods for solving the Black Scholes Partial differential equation are the Explicit method, the Fully Implicit method and the Crank-Nicolson method. These are closely related but differ in stability, accuracy and execution speed.

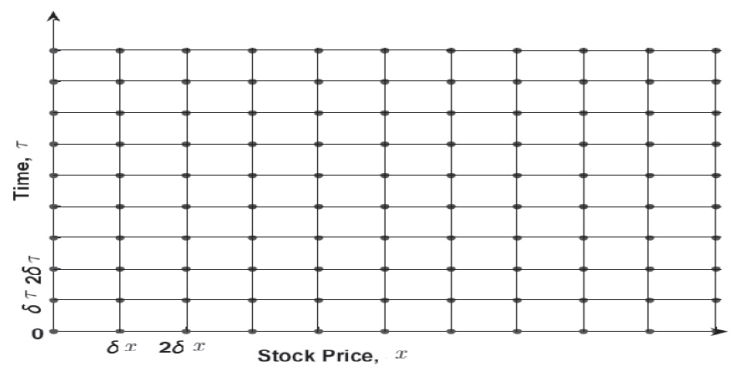

(a) The mesh points for the finite difference approximation.

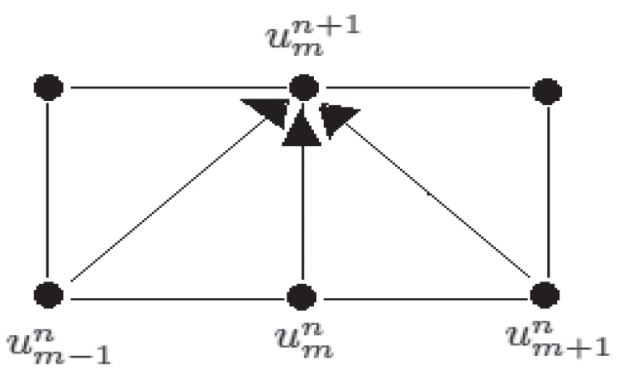

(b) Explicit Finite Difference Method

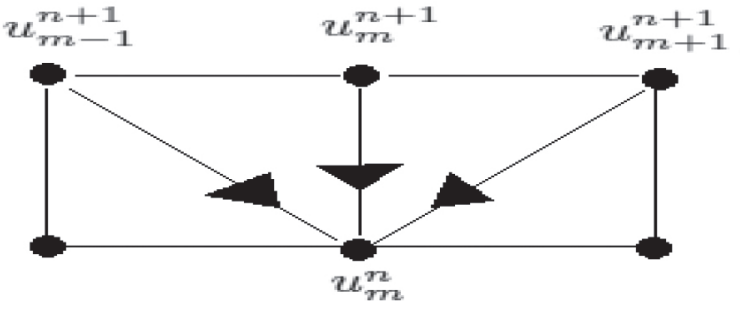

(c) Fully-implicit Finite Difference Method

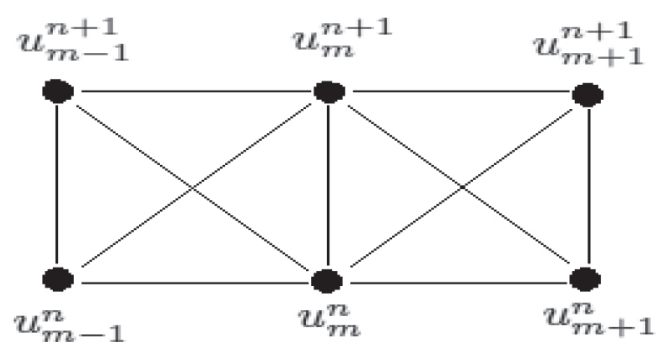

(d) Crank Nicolson Fïnite Difference Method

Figure 2: Finite Difference

\section{Discretization of the Equation}

We divide the $(x, \tau)$ plane into a regular finite mesh, and take finite-difference approximations of the linear complementarity form problem. We approximate the terms $\partial u / \partial \tau-\partial^{2} u / \partial^{2} x$ by the finite differences on a regular mesh with step sizes $\delta \tau$ and $\delta x$, and we truncate so that $x$ lies between $M \delta x$ and $M^{+} \delta x$, where $M$ is a large negative number and $M^{+}$is a large positive number. We divide the non-dimensional time to expiry of the option, $\frac{1}{2} \sigma^{2} T$, into $\mathrm{N}$ equal time-steps so that $\delta \tau=\frac{1}{2} \sigma^{2} T / N$.

The idea of finite difference methods is to replace the partial derivatives in the equation by their differencequotient approximations based on Taylor series expansions of functions near the points of interest, and then let the computer solve the resulting differential equation. We divide the $x$-axis into equally spaced nodes a distance $\delta x$ apart, and the $\tau$-axis into equally spaced nodes a distance $\delta t$ apart. This divides the $(x, \tau)$ plane into a mesh, where the mesh points have the form $(m \delta x, n \delta \tau)$, $M \leq x \leq M^{+}$and $0<n \leq N$. We write $u_{m}^{n}=u(m \delta x, n \delta \tau)$ as the value of $u(x, \tau)$ at the mesh point $(m \delta x, n \delta \tau)$, and $g_{m}{ }^{n}=g(m \delta x, n \delta \tau)$.

\section{Explicit Method}

Given that we know the value of an option at the maturity time, it is possible to give an expression that gives us the next value $u_{m}^{n+1}$ Explicit in terms of the given values $u_{m-1}^{n}, u_{m}^{n}, u_{m+1}^{n}$.

Using a forward difference to approximate $\partial u / \partial \tau$, and a second-order central difference for $\partial^{2} u / \partial x^{2}$, we can rewrite the diffusion equation (26) as

$\frac{u_{m}^{n+1}-u_{m}^{n}}{\delta \tau}+O(\delta \tau)=\frac{u_{m+1}^{n}-2 u_{m}^{n}+u_{m-1}^{n}}{(\delta x)^{2}}+O\left((\delta x)^{2}\right)$

If we ignore terms of $O(\delta x)$ and $O\left((\delta x)^{2}\right)$, we can rearrange (28) to give the difference equation

$$
u_{m}^{n+1}=\alpha u_{m+1}^{n}+(1-2 \alpha) u_{m}^{n}+\alpha u_{m-1}^{n}
$$


where $\alpha=\delta \tau /(\delta x)^{2}$. At time step $n+1$, we already know the values of $u_{m}^{n}$ for all $\mathrm{n}$ so we can explicitly calculate $u_{m}^{n+1}$. After we calculate $u_{m}^{n+1}$ using (29), we also need the equation to satisfy the constraint $u(x, \tau)-g(x, \tau) \geq 0$. Thus the scheme for this explicit method can be expressed as:

$$
\begin{gathered}
y_{m}^{n+1}=\alpha u_{m-1}^{m}+(1-2 \alpha) u_{m}^{n}+\alpha u_{m+1}^{n} ; \\
u_{m}^{n+1}=\max \left(y_{m}^{n+1}, g_{m}^{n+1}\right)
\end{gathered}
$$

With this approach, the stability question arises. The system (29) is stable if $0<\alpha \leq 1 / 2$ and unstable if $\alpha>$ $1 / 2^{6}$, which puts severe constraints on the size of time steps. Hence there is need to consider a more stable method, such as the fully-implicit method. The implicit finite-difference method is stable for any $\alpha>0$, which suggests that we can solve the diffusion equation with larger time-steps using an implicit algorithm than we can using an explicit algorithm.

\section{Fully-Implicit Method}

Given that we know the value of an option at the maturity time, it is possible to give an expression that gives us the next value $u_{m}^{n}$ implicit in terms of the given values

$$
u_{m-1}^{n+1}, u_{m}^{n+1}, u_{m+1}^{n+1} \text {. }
$$

Using a backward difference to approximate $\partial u / \partial \tau$, and a second-order central difference for $\partial^{2} u / \partial x^{2}$, we can rewrite the diffusion equation (26) as

$\frac{u_{m}^{n+1}-v_{m}^{n}}{\delta \tau}+O(\delta \tau)=\frac{u_{m+1}^{n+1}-2 u_{m}^{n+1}+u_{m-1}^{n+1}}{(\delta x)^{2}}+O\left((\delta x)^{2}\right)$

If we ignore terms of $O(\delta x)$ and $O\left((\delta x)^{2}\right)$, we can rearrange (31) to give the difference equation

$$
u_{m}^{n}=-\alpha u_{m-1}^{n+1}+(1+2 \alpha) u_{m}^{n+1}-\alpha u_{m+1}^{n+1}
$$

The new values cannot be separated out immediately and solved for explicitly in terms of the old values. This is the reason it is called the implicit scheme. The linear complementarity problem (23) is then approximated by

$$
\begin{gathered}
\left.-\alpha u_{m-1}^{n+1}+(1+2 \alpha) u_{m}^{n+1}-\alpha u_{m+1}^{n+1}-u_{m}^{n}\right) \\
\left(u_{m}^{n+1}-g_{m}^{n+1}\right)=0
\end{gathered}
$$

at time step $n+1$.

Let

$$
\begin{array}{r}
u^{n}=\left[\begin{array}{c}
u_{M^{-}+1}^{n} \\
u_{M^{-}+2}^{n} \\
\vdots \\
u_{M^{+-2}}^{n} \\
u_{M^{+}-1}^{n}
\end{array}\right], g^{n}=\left[\begin{array}{c}
g_{M^{-}+1}^{n} \\
g_{M^{-}+2}^{n} \\
\vdots \\
g_{M^{+}-2}^{n} \\
g_{M^{+}-1}^{n}
\end{array}\right] \\
b^{n}=\left[\begin{array}{c}
b_{M^{-}+1}^{n} \\
b_{M^{-}+2}^{n-1} \\
\vdots \\
b_{M^{+-2}}^{n} \\
b_{M^{+}-1}^{n}
\end{array}\right]=\left[\begin{array}{c}
u_{M^{-}+1}^{n} \\
u_{M^{-}+2}^{n-1} \\
\vdots \\
0 \\
u_{M^{+-1}}^{n-1} \\
u_{M^{+-1}}^{n-1}
\end{array}\right]+\alpha\left[\begin{array}{c} 
\\
\vdots \\
0 \\
g_{M^{+}}^{n}
\end{array}\right]
\end{array}
$$

and the coefficient matrix

$$
C=\left[\begin{array}{ccccc}
(1+2 \alpha) & -\alpha & 0 & \ldots & 0 \\
-\alpha & (1+2 \alpha) & \ddots & \ddots & \ldots \\
0 & -\alpha & \ddots & \ddots & \ldots \\
\vdots & \ddots & \ddots & \ddots & -\alpha \\
0 & \ldots & 0 & -\alpha & (1+2 \alpha)
\end{array}\right]
$$

We want to solve the following constrained matrix problem

$$
\text { Cun+1 }=b n+1
$$

and check if the $u \geq g$ constraint is satisfied. To solve equation (37), observe that $\mathrm{C}$ is an invertible matrix due to the Gerschgorin theorem. Therefore, equation (37) has a solution.

\section{Theorem (GERSCHGORIN)}

Let $A=\left(a_{i, j}\right)$ be an arbitrary $m \times m$ complex matrix, and let

$$
\Lambda_{i} \equiv \Sigma_{j=1, j \neq i}^{m}\left|a_{i, j}\right|, \quad 1 \leq i \leq m .
$$

Then, all the eigenvalues $\lambda$ of $A$ lie in the union of the disks

$$
\left|z-a_{i, j}\right| \leq \Lambda_{i}, \quad 1 \leq i \leq m .
$$

Observe that the matrix $\mathrm{C}$ is symmetric so all of the eigenvalues are real. Then, let $a_{i, j}=1+2 \alpha$. For $\mathrm{i}=1$ and $\mathrm{m}, \Lambda_{i}=\alpha$. Then $z \geq a_{i, j}-\Lambda_{i}=1+\alpha$ so for $\alpha \geq 0$, $\mathrm{z}$ is always positive. For $2 \leq i \leq m-1, \Lambda_{1}=(\alpha)+(\alpha)=2 \alpha$. Then $z \geq a_{i, j}-\Lambda_{i}=1$. Thus, for $\alpha \geq 0$, all the eigenvalues of $\mathrm{C}$ are positive real numbers, suggesting $C$ is invertible and we can rewrite equation (37) as

$$
u^{n+1}=C^{-1} b^{n+1} \text {. }
$$

We can first form $b^{n+1}$ from $u^{n}$ and the boundary conditions. Using the known initial condition $u^{0}$, we can obtain $u^{n+1}$ sequentially using an iterative method such as Projected SOR.

\section{Projected SOR Algorithm}

Projected SOR is a minor modification of the SOR method, Successive Over-Relaxation. The SOR method is used to speed up convergence of iterations. The projected SOR algorithm involves five steps. The first step is to rewrite equation (32) as:

$$
u_{m}^{n+1}=\frac{1}{1+2 \alpha}\left(b_{m}^{n+1}+\alpha\left(u_{m-1}^{n+1}+u_{m+1}^{n+1}\right)\right),
$$

where $M+1 \leq m \leq M^{+}-1$.

Let $u_{m}^{n, k}$ be the $\mathrm{k}$-th iterate for $u_{m}^{n}$. Let us denote the initial guess by $u_{m}^{n, 0}$. As $k \rightarrow \infty$, we expect $u_{m}^{n, k} \longrightarrow u_{m}^{n}$. We update the values as soon as they are available and add a correction term $u_{m}^{n, k+1}-u_{m}^{n, k}$ to the original $u_{m}^{n, k+1}$. We also incorporate an over-correction or overrelaxation parameter $\omega$, which has optimal values between 1 and 2, and finally, we take the maximum between the estimated $u_{m}^{n+1, k}$ and the payoff $g_{m}^{n+1}$. The fully-implicit scheme is thus expressed as, 


$$
\begin{gathered}
y_{m}^{n+1, k+1}=\frac{1}{1+2 \alpha}\left(b_{m}^{n+1}+\alpha\left(u_{m-1}^{n+1, k+1}+u_{m+1}^{n+1, k}\right)\right) \\
u_{m}^{n+1, k+1}=\max \left(u_{m}^{n+1, k}+\omega\left(y_{m}^{n+1, k+1}-u_{m}^{n+1, k}\right), g_{m}^{n+1}\right)
\end{gathered}
$$

Until the difference between $u_{m}^{n+1, k+1}$ and $u_{m}^{n+1, k}$ is small enough to be ignored, we set $u_{m}^{n+1}=u_{m}^{n+1, k+1}$.

\section{The Crank-Nicolson Method}

The Crank Nicolson finite difference method is the average of the implicit and explicit methods and hence may improve accuracy. In this method, six neighboring option values are interrelated in the following way:

One improvement the Crank-Nicolson Method has over the fully-implicit method is that it increases the temporal convergence rate from $O(\delta \tau)$ to $O\left(\left((\delta \tau)^{2}\right)\right)$. The equation of the scheme is

$$
\begin{aligned}
& u_{m}^{n+1}-\frac{1}{2} \alpha\left(u_{m-1}^{n+1}-2 u_{m}^{n+1}+u_{m+1}^{n+1}\right) \\
& =u_{m}^{n}+\frac{1}{2} \alpha\left(u_{m-1}^{n}-2 u_{m}^{n}+u_{m+1}^{n}\right),
\end{aligned}
$$

where $\alpha=\delta \operatorname{tau} /(\delta x)^{2}$.

$$
\begin{aligned}
& (1+\alpha) u_{m}^{n+1}-\frac{1}{2} \alpha\left(u_{m-1}^{n+1}+u_{m+1}^{n+1}\right) \\
& =(1-\alpha) u_{m}^{n}+\frac{1}{2} \alpha\left(u_{m-1}^{n}+u_{m+1}^{n}\right)
\end{aligned}
$$

Let $Z_{m}^{n}=(1-\alpha) u_{m}^{n}+\frac{1}{2} \alpha\left(u_{m-1}^{n}+u_{m+1}^{n}\right)$,

then

$$
(1+\alpha) u_{m}^{n+1}-\frac{1}{2} \alpha\left(u_{m-1}^{n+1}+u_{m+1}^{n+1}\right)=Z_{m}^{n} .
$$

There remains the problem of finding the $u_{m}^{n}$ for $n \geq 1$ and $M<m<M^{+}$from (44). We can write the problem as a linear system

$$
C u^{n+1}=b-
$$

Define a new matrix by

$$
b^{n}=\left[\begin{array}{c}
b_{M^{-}+1}^{n} \\
b_{M^{-}+2}^{n} \\
\vdots \\
b_{M^{+-2}}^{n} \\
b_{M^{+-1}}^{n}
\end{array}\right]=\left[\begin{array}{c}
Z_{M^{-}+1}^{n} \\
Z_{M^{-}+2}^{n} \\
\vdots \\
Z_{M^{+-2}}^{n} \\
Z_{M^{+-1}}^{n}
\end{array}\right]+\frac{1}{2} \alpha\left[\begin{array}{c}
g_{M^{-}}^{n+1} \\
0 \\
\vdots \\
0 \\
g_{M^{+}}^{n+1}
\end{array}\right]
$$

The tri-diagonal symmetric matrix $\mathrm{C}$ becomes

$$
C=\left[\begin{array}{ccccc}
(1+\alpha) & -\frac{1}{2} \alpha & 0 & \ldots & 0 \\
-\frac{1}{2} \alpha & (1+\alpha) & \ddots & \ddots & \ldots \\
0 & -\frac{1}{2} \alpha & \ddots & \ddots & \ldots \\
\vdots & \ddots & \ddots & \ddots & -\frac{1}{2} \alpha \\
0 & \ldots & 0 & -\frac{1}{2} \alpha & (1+\alpha)
\end{array}\right] .
$$

and the coefficient matrix

$$
u^{n+1}=\left[\begin{array}{c}
u_{M-1}^{n+1} \\
u_{M^{-}+2}^{n+1} \\
\vdots \\
u_{M^{+}-2}^{n+1} \\
u_{M^{+}-1}^{n+1}
\end{array}\right]
$$

Using Theorem (Gerschgorin), we see that the matrix $\mathrm{C}$ is invertible. Similar to the fully-implicit method, we adopt the projected SOR algorithm and obtain

$$
\begin{gathered}
y_{m}^{n+1, k+1}=\frac{1}{1+\alpha}\left(b_{m}^{n}+\frac{1}{2} \alpha\left(u_{m-1}^{n+1, k+1}+u_{m+1}^{n+1, k}\right)\right) \\
u_{m}^{n+1, k+1}=\max \left(u_{m}^{n+1, k}+\omega\left(y_{m}^{n+1, k+1}-u_{m}^{n+1, k}\right), g_{m}^{n+1}\right)
\end{gathered}
$$

Until the difference between $u_{m}^{n+1, k+1}$ and $u_{m}^{n+1, k}$ is small enough to be ignored, we set $u_{m}^{n+1}=u_{m}^{n+1, k+1}$.

\section{NUMERICAL RESULTS}

The following results are obtained through implementing the three finite difference algorithms described in the previous sections in MATLAB. Table 1 and Table 3 show that the three finite difference methods provide us similar result. One limitation for the explicit scheme is stable only when the ratio of the time step to the square of the space step is not greater than $1 / 2$, which imposes restrictions on the number of necessary time steps. Though the explicit method is relatively easy to implement, the fully-implicit and the Crank Nicolson methods have better stability properties. Besides comparing the results of the three methods, we are also interested in the effects of dividend payments on option pricing. Table 2 and Table 4 suggests that as dividend rate increases, call option value decreases. A firm's dividend payout policy affects option values because a high dividend payment decreases the rate of growth of the asset price. Thus there is a lower expected rate of capital gain, leading to a lower potential payoff. In addition, since the cash dividend is received by whoever owns the stock until the ex-dividend date, holders might exercise the option just prior to the ex-dividend date. Exercising prior to the ex-dividend date is always optimal when the dividend payment is large. Early exercise will be likely to happen only if the asset is expected to pay a dividend prior the expiration date.

\section{European Option}

Table 1

\begin{tabular}{|c|c|c|c|}
\hline S & Explicit & Fully-Implicit & Crank-Nicolson \\
\hline 3 & 0.0047 & 0.0048 & 0.0048 \\
\hline 5 & 0.1490 & 0.1486 & 0.1492 \\
\hline 7 & 0.7409 & 0.7389 & 0.7404 \\
\hline 9 & 1.8383 & 1.8362 & 1.8377 \\
\hline 11 & 3.2914 & 3.2902 & 3.2912 \\
\hline
\end{tabular}

A comparison of the option values of the three methods at $\mathrm{E}=8, \mathrm{~d}=0.08, \mathrm{r}=0.1, \sigma=0.4$ and $\mathrm{T}=1$. 
Table 2

\begin{tabular}{|c|c|c|c|c|c|}
\hline $\mathrm{S}$ & $\mathrm{d}=0.03$ & $\mathrm{~d}=0.05$ & $\mathrm{~d}=0.08$ & $\mathrm{~d}=0.11$ & $\mathrm{~d}=0.13$ \\
\hline 3 & 0.0072 & 0.0061 & 0.0 .0048 & 0.0 .0034 & 0.0029 \\
\hline 5 & 0.1946 & 0.1749 & 0.1486 & 0.1220 & 0.1089 \\
\hline 7 & 0.9017 & 0.8336 & 0.7389 & 0.6466 & 0.5945 \\
\hline 9 & 2.1458 & 2.0180 & 1.8362 & 1.6606 & 1.5548 \\
\hline 11 & 3.7409 & 3.5562 & 3.2902 & 3.0336 & 2.8737 \\
\hline
\end{tabular}

A comparison of the option values of the three methods at $\mathrm{E}=8, \mathrm{~d}=0.08, \mathrm{r}=0.1, \sigma=0.4$ and $\mathrm{T}=1$.

\section{American Option}

Table 3

\begin{tabular}{|c|c|c|c|}
\hline $\mathrm{S}$ & Explicit & Fully-Implicit & Crank-Nicolson \\
\hline 3 & 0.0047 & 0.0049 & 0.0048 \\
\hline 5 & 0.1496 & 0.1492 & 0.1492 \\
\hline 7 & 0.7473 & 0.7402 & 0.7404 \\
\hline 9 & 1.8664 & 1.8389 & 1.8378 \\
\hline 11 & 3.3707 & 3.2975 & 3.2923 \\
\hline
\end{tabular}

A comparison of the option values of the three methods at $\mathrm{E}=8, \mathrm{~d}=0.08, \mathrm{r}=0.1, \sigma=0.4$ and $\mathrm{T}=1$.

Table 4

\begin{tabular}{|c|c|c|c|c|c|}
\hline $\mathrm{S}$ & $\mathrm{d}=0.03$ & $\mathrm{~d}=0.05$ & $\mathrm{~d}=0.08$ & $\mathrm{~d}=0.11$ & $\mathrm{~d}=0.13$ \\
\hline 3 & 0.0073 & 0.0063 & 0.0047 & 0.0039 & 0.0033 \\
\hline 5 & 0.1956 & 0.1758 & 0.1492 & 0.1263 & 0.1128 \\
\hline 7 & 0.9031 & 0.8348 & 0.7402 & 0.6550 & 0.6048 \\
\hline 9 & 2.1473 & 2.0193 & 1.8389 & 1.6789 & 1.5915 \\
\hline 11 & 3.7425 & 3.5577 & 3.2975 & 3.0911 & 2.9990 \\
\hline
\end{tabular}

A comparison of the option values of the three methods at $\mathrm{E}=8, \mathrm{~d}=0.08, \mathrm{r}=0.1, \sigma=0.4$ and $\mathrm{T}=1$.

\section{CONCLUSION}

Option pricing has been an increasingly popular field to study. Options provide investors limited downside in speculative trading and enable investors to hedge and to minimize risk. The value of options depends on a number of variables such as stock price $S$, exercise price $E$, Volatility $\sigma$, time to expiration $T$, interest rate $r$ and dividend payments $D_{0}$. The study of European (American) call options with dividends with transformations to a usual (linear complementarity) form. With three finite difference methods to solve the option valuation problem of European (American) call with dividends and implements the methods in MATLAB to obtain numerical results that gauge the potential for computing based on the usual (linear complementarity) form. Through the numerical simulations of the model problem, we also verify that the explicit method suffers from the limitation on the number of time steps size and the both fullyimplicit and Crank-Nicolson method are unconditionally stable and Crank Nicolson method converge faster than the explicit and implicit method. We see from the numerical values and financial theories that there is a negative relationship between dividend payouts and call option values. Option holders must take into consideration the effect of dividend when deciding if the option should be early exercised.

\section{ACKNOWLEDGMENT}

I would like to express my sincere gratitude to my cosupervisor Prof. Dr. Harihar Khanal, Department of Mathematics, Embry-Riddle Aeronautical University, USA.

\section{REFERENCES}

1. Black, F.and Scholes, M. 1973, The pricing of options and corporate liabilities, J. Polit. Econ. 81: $637-659$.

2. Bhowmik, S. K. 2010. Stable numerical schemes for a partly convolutional partial integrodifferential equation. Applied Mathematics and Computation. 217(8) : 42174226.

3. Merton, R.C. 1973, Theory of rational option pricing, Bell J. Econ., Vol. 4, No. 1: $141-183$.

4. Tavella, D. and Randall, C. 2000. Pricing Financial Instruments: The Finite Difference Method. John Wiley and Sons, New York.

5. Wilmott, P., Howison, S. and Dewynne, J. 2002. The Mathematics of Financial Derivatives, A Student Introduction, Cambridge University Press.

6. Wilmott, P., Howison, S. and Dewynne, J. 1995. The Mathematics of Financial Derivatives. A Student introduction. Cambridge University Press, Cambridge. 